This is a self-archived version of an original article. This version may differ from the original in pagination and typographic details.

Author(s): Heikkilä, Tero; Ojajärvi, Risto; Maasilta, Ilari; Strambini, E.; Giazotto, F.; Bergeret, F.S.

Title: Thermoelectric Radiation Detector Based on Superconductor-Ferromagnet Systems

Year: 2018

Version: Published version

Copyright: @ 2018 American Physical Society

Rights: In Copyright

Rights url: http://rightsstatements.org/page//nC/1.0/?language=en

Please cite the original version:

Heikkilä, T., Ojajärvi, R., Maasilta, I., Strambini, E., Giazotto, F., \& Bergeret, F. S. (2018).

Thermoelectric Radiation Detector Based on Superconductor-Ferromagnet Systems. Physical

Review Applied, 10(3), Article 034053. https://doi.org/10.1103/PhysRevApplied.10.034053 


\title{
Thermoelectric Radiation Detector Based on Superconductor-Ferromagnet Systems
}

\author{
T. T. Heikkilä, ${ }^{1,}{ }^{*}$ R. Ojajärvi, ${ }^{1}$ I. J. Maasilta, ${ }^{1}$ E. Strambini, ${ }^{2}$ F. Giazotto, ${ }^{2}$ and F. S. Bergeret ${ }^{3,4}$ \\ ${ }^{1}$ Department of Physics and Nanoscience Center, University of Jyvaskyla, P.O. Box 35, \\ FI-40014 University of Jyväskylä, Finland \\ ${ }^{2}$ NEST, Istituto Nanoscienze-CNR and Scuola Normale Superiore, 56127 Pisa, Italy \\ ${ }^{3}$ Centro de Física de Materiales, Centro Mixto CSIC-UPV/EHU, Paseo Manuel de Lardizabal 5 , \\ 20018 San Sebastián, Spain \\ ${ }^{4}$ Donostia International Physics Center, Paseo Manuel de Lardizabal 4, 20018 San Sebastián, Spain
}

(Received 29 September 2017; revised manuscript received 23 April 2018; published 25 September 2018)

\begin{abstract}
We suggest an ultrasensitive detector of electromagnetic fields exploiting the giant thermoelectric effect recently found in superconductor-ferromagnet hybrid structures. Compared with other types of superconducting detectors where the detected signal is based on variations of the detector impedance, the thermoelectric detector has the advantage of requiring no external driving fields. This is especially relevant in multipixel detectors, where the number of bias lines and the heating induced by them are an issue. We propose different material combinations to implement the detector and provide a detailed analysis of its sensitivity and speed. In particular, we perform a proper noise analysis that includes the cross correlation between heat and charge current noise and thereby describes also thermoelectric detectors with a large thermoelectric figure of merit.
\end{abstract}

DOI: 10.1103/PhysRevApplied.10.034053

\section{INTRODUCTION}

Some of the most accurate sensors of wideband electromagnetic radiation are based on superconducting films. Such sensors, in particular the transition-edge sensor (TES), are used in a wide variety of applications requiring extremely high sensitivity. Those applications include detection of the cosmic microwave background [1-3] and other tasks in astrophysics [4], generic-purpose terahertzradiation sensing used, for example, in security imaging [5], gamma-ray spectroscopy of nuclear materials [6], and analysis of materials by detection of fluorescent $\mathrm{x}$-rays excited by ion beams [7], short laser-driven x-ray pulses [8], or syncrotrons [6]. Many of these applications would benefit from the addition of more pixels (i.e., more sensors) to increase the collection efficiency, detection bandwidth, or the spatial or angular resolution. However, operating large arrays of TESs can be problematic as each pixel requires a bias line. This can be cumbersome in the presence of thousands of pixels, even with advanced multiplexing techniques [6]. Moreover, the bias lines tend to carry heat into the system especially by radiation, reducing, for example, its overall noise performance. In addition, a TES always dissipates power at the pixel, giving constraints on the cryogenic design for large arrays. One alternative

\footnotetext{
*tero.t.heikkila@jyu.fi
}

is the kinetic inductance detector (KID) [9-11] and its variants [12,13], the most common being the type with passive frequency-domain multiplexing using superconducting microwave resonators [14]. With such a device, a single pair of coaxial cables can be used to probe a large array of pixels, but the probe power is, by necessity, also partially dissipated at the detectors.

Both the TES and the KID are based on the measurement of an impedance of the sensor (i.e., response to a probe signal). It would generally be beneficial if one could get rid of the probe signal altogether, so that the measured signal would result directly from the radiation coupled to the detector. This is what happens in thermoelectric detection [15-17], where the temperature rise caused by the absorption of radiation is converted into an electric voltage or current that can then be detected. Such thermoelectric detectors have been discussed before, but they have not been considered for ultrasensitive low-temperature detectors for the simple reason that thermoelectric effects are typically extremely weak at low temperatures. On the other hand, at high temperatures, where such thermoelectric effects would be strong enough, the thermal noise hampers the device sensitivity.

We suggest overcoming these problems in a superconductor-ferromagnet thermoelectric detector (SFTED) [18] by exploiting the newly discovered giant thermoelectric effect that occurs in superconductor- 
ferromagnet heterostructures [19-22] for radiation sensing. As this thermoelectric effect can be realized with close to Carnot efficiency $[19,22]$ even at subkelvin temperatures, the resulting detector can have a large signal-to-noise ratio, and a noise-equivalent power (NEP) rivaling those of the best TESs and KIDs without the burden of having to use additional bias lines for probing the sensor, and with zero (for ideal amplification) or at most very small nonsignal power dissipation at the sensor location. The only part of the system where external power is needed is in the detection of the thermoelectric currents (i.e., at the amplifier), which can be taken far from the active sensing region.

Recently the use of superconductor-ferromagnet structures in thermometry has been discussed [23]. Despite some similarities, thermometers and radiation detectors have quite different requirements regarding their sensitivity. In particular, the sensitivity of radiation detectors is typically dictated by the temperature-fluctuation noise, which is not an issue as such for thermometers. In this paper we concentrate on finding and optimizing the relevant figures of merit for radiation detection, and hence we cannot benefit much from the results in thermometry.

For concreteness, we consider the detector realization depicted in Fig. 1. The sensor element (i.e., one pixel of a possible detector array) is formed from a thin-film superconductor-ferromagnetic insulator bilayer coupled to superconducting antennas via a clean (Andreev) contact. This bilayer is further connected, via a tunnel junction (magnetic or normal) to a ferromagnetic electrode [24]. The current injected into the ferromagnetic electrode or the voltage generated across the tunnel junction is detected by a superconducting-quantum-interference-device (SQUID) current amplifier or a field-effect transistor, respectively. In what follows, we describe conditions for measuring radiation in the far-IR region, in which case it can be coupled to the detector via antennas. Alternatively, the detector could be used for measuring radiation at higher frequencies (such as x-ray frequencies), in which case the system should be connected to an additional, larger absorber element.

We consider the radiation to be directed to the detector via a superconducting antenna (not shown in Fig. 1) that is coupled via a clean (Andreev) contact to the active superconductor-ferromagnetic insulator region (absorber). To prevent heat leaking from the absorber, the superconductor used in the antenna should be fabricated from a material with a higher superconducting gap $\Delta_{A}$ than the one used in the absorber, $\Delta$. One possible combination could be a $\mathrm{Nb}$ antenna and an $\mathrm{Al}$ absorber. For optimal quantum efficiency, the normal-state resistance of the absorber (seen by radiation at frequencies higher than $\Delta / h$, where $h$ is Planck's constant) should be matched to the specific impedance of the antenna, typically somewhat below the vacuum impedance. For an Al film thickness of $10 \mathrm{~nm}$, a typical sheet resistance is $5-10 \Omega$. Hence, a

\section{(a)}

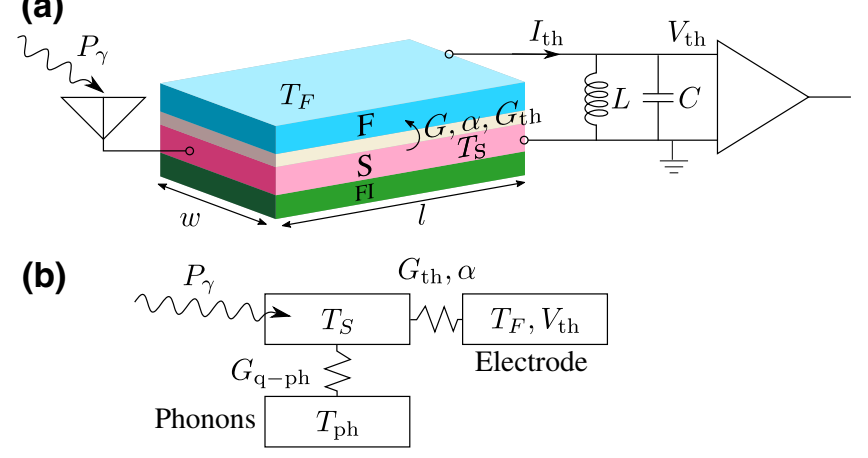

FIG. 1. (a) The thermoelectric detector, where a temperature difference $T_{S}-T_{F}$ drives a thermoelectric current $I_{\text {th }}$ and/or a thermovoltage $V_{\text {th }}$ across a spin-polarized junction. The latter is composed of either a normal insulator and a ferromagnetic electrode $(\mathrm{F})$ or a ferromagnetic insulator (FI) and a normal metal electrode. (b) Heat balance: incoming radiation power heats up the quasiparticles in the spin-split superconductor (S), and the amount of heating depends on the heat conductances to the main heat baths.

$1-\mu \mathrm{m}$-wide film with length $l=10 \mu \mathrm{m}$ would have resistance $R_{\gamma}=50-100 \Omega$ seen by the radiation, thereby matching well with typical antennas. In what follows, we hence choose an absorber region of this size. Reducing (increasing) the width and length while keeping their ratio constant would result in the same quantum efficiency but decreased (increased) noise and dynamic range.

The absorber superconductor is placed in contact with a ferromagnetic insulator that exerts a magnetic proximity effect on the former, resulting in a spin-splitting exchange field $h$ inside the superconductor. In Al, large induced spinsplitting fields have been detected by contacting it, for example, with EuS [25] or EuO [26]. At low temperatures compared with the superconductor critical temperature $T_{c}$, the exchange field does not have a major effect on the order parameter $\Delta[27,28]$. However, it results in a strong (and opposite) electron-hole asymmetry in each spin component in the direction specified by the magnetization of the ferromagnetic insulator. This asymmetry can be used to generate a thermoelectric signal if the superconductor is connected via a spin filter to another electrode $[19,22]$. This spin filtering is provided here by the ferromagnetic electrode and is quantified by the normal-state spin polarization $P=\left(G_{\uparrow}-G_{\downarrow}\right) /\left(G_{\uparrow}+G_{\downarrow}\right) \in[-1,1]$, where $G_{\sigma}$ is the normal-state conductance of the superconductorferromagnet contact for spin channel $\sigma$. In what follows, we also characterize this contact via its spin-averaged normal-state conductance $G_{T}$. In practice, oxide contacts with ferromagnetic metals such as Ni, Co, or Fe have $P \sim$ $0.1-0.45[29,30]$, whereas use of ferromagnetic insulator contacts may lead to polarizations exceeding $P \sim 0.9$ [31]. The precise value of $G_{T}$ for a given area of the junction can be controlled by the thickness of the tunnel junction. 


\section{NOISE-EQUIVALENT POWER OF A THERMOELECTRIC DETECTOR}

We first consider a generic thermoelectric element working as a radiation sensor and analyze its figures of merit, in particular the NEP and the thermal time constant $\tau_{T}$, both defined in detail below. As the radiation with power $P_{\gamma}$ is absorbed in the absorber, it first creates a strong nonequilibrium state of the quasiparticles. This nonequilibrium state relaxes via (i) quasiparticle-quasiparticle collisions, (ii) spurious processes such as quasiparticle-phonon relaxation, and (iii) the escape of the quasiparticles to the (ferromagnetic) electrode. The last process yields the detected signal. Moreover, some of the excitations may escape as quasiparticles to the antenna. We assume that relaxation via quasiparticle-quasiparticle collisions dominates so that the quasiparticles thermalize between themselves before escaping to the antenna, and therefore in what follows we disregard escape of excitations as quasiparticles to the antenna. As a result of this chain of events, the quasiparticles in the absorber heat up to temperature $T_{S}=T+\Delta T$ determined from a heat-balance equation [32]:

$$
C_{h} \frac{d \Delta T}{d t}=P_{\gamma}-G_{\mathrm{th}}^{\mathrm{tot}} \Delta T+\alpha V_{\mathrm{th}},
$$

where $C_{h}$ is the heat capacity of the absorber and $G_{\mathrm{th}}^{\text {tot }}=$ $G_{q \text {-ph }}+G_{\text {th }}$, where $G_{q \text {-ph }}$ and $G_{\text {th }}$ denote the heat conductances from quasiparticles to the phonons and to the ferromagnetic electrode, respectively. In the linear regime we assume that both reside at the bath temperature $T$. The last term results from the Peltier heat current driven by the induced thermovoltage across the superconductorferromagnet junction, and it is proportional to the temperature difference $\Delta T$. We assume that the detector operates at low powers $P_{\gamma}$ so that these linear response relations are sufficient. The detector characteristics depend strongly on the chosen $T$.

The induced temperature difference (in the frequency domain $) \Delta T=\left(P_{\gamma}+\alpha V_{\text {th }}\right) /\left(i \omega C_{h}+G_{q \text {-ph }}+G_{\text {th }}\right)$ drives a thermoelectric current $I_{\text {th }}=\alpha \Delta T / T-G V_{\text {th }}$ into the ferromagnet and ultimately to an amplifier. To focus on detector performance limits first, we disregard the backaction noise from the amplifier, and consider the amplifier only as a reactive element: either a capacitor or an inductor, corresponding to a field-effect transistor or SQUID amplifier, respectively. Therefore, the thermoelectric current equals $V_{\text {th }}[i \omega C+1 /(i \omega L)]$ across the amplifier with capacitance $C$ and inductance $L$ in parallel. One can obtain the practical limits of voltage (current) measurements by considering $\omega \neq 0$ and taking the limit $L \rightarrow \infty(C \rightarrow \infty)$. From these relations we can obtain the voltage and current responsivities:

$$
\lambda_{V} \equiv \frac{V_{\text {th }}}{P_{\gamma}}=\frac{\alpha}{Y_{\text {th }}^{\text {tot }} Y^{\text {tot }} T-\alpha^{2}}, \quad \lambda_{I} \equiv \frac{I_{L}}{P_{\gamma}}=\frac{\lambda_{V}}{i \omega L},
$$

where $I_{L}$ is the current across the inductor. The relevant responsivity depends on the choice of the amplifier. Here $Y_{\text {th }}^{\text {tot }}=i \omega C_{h}+G_{\text {th }}^{\text {tot }}$ and $Y^{\text {tot }}=G+i \omega C+1 /(i \omega L)$ are the thermal and electrical admittances, respectively. Note that $Z T(\omega)=\alpha \lambda_{V}$ is a finite-frequency generalization of the usual thermoelectric figure of merit.

We next consider the temperature fluctuation $\delta T$, voltage noise $\Delta V$ across the capacitor, and current noise $\Delta I_{L}$ across the inductor. These are driven by the three intrinsic noise sources: the charge and heat current noises $\delta I$ and $\delta \dot{Q}_{J}$ across the thermoelectric junction and the heat current noise $\delta \dot{Q}_{q \text {-ph }}$ for the quasiparticle-phonon process. The heat balance equation and the Kirchoff law for the noise terms read

$$
\begin{gathered}
Y_{\text {th }}^{\mathrm{tot}} \delta T=\delta \dot{Q}_{q \text {-ph }}+\delta \dot{Q}_{J}+\alpha \Delta V, \\
Y^{\mathrm{tot}} \Delta V=\delta I+\alpha \delta T / T .
\end{gathered}
$$

Solving these yields

$$
\Delta V=\lambda_{V}\left(\delta \dot{Q}_{q-\mathrm{ph}}+\delta \dot{Q}_{J}+Y_{\mathrm{th}}^{\mathrm{tot}} T \delta I / \alpha\right)
$$

and $\Delta I_{L}=\Delta V /(i \omega L)$. To find the second-order correlator of these noise terms, we assume that the intrinsic correlators satisfy

$$
\begin{gathered}
\left\langle\delta I^{2}\right\rangle=4 k_{B} T G, \\
\left\langle\delta \dot{Q}_{J}^{2}\right\rangle=4 k_{B} T^{2} G_{\mathrm{th}}, \\
\left\langle\delta I \delta \dot{Q}_{J}\right\rangle=-4 k_{B} T \alpha, \\
\left\langle\delta \dot{Q}_{q-\mathrm{ph}}^{2}\right\rangle=4 k_{B} T^{2} G_{q-\mathrm{ph}} .
\end{gathered}
$$

These result from the fluctuation-dissipation theorem for the individual contacts. In particular, the cross-noise term is important for strong thermoelectric response, and was not taken into account before; it was, for example, disregarded in Ref. [16]. The total voltage-noise spectral density is (note that this is the symmetrized voltage-noise correlator, and therefore one needs to take the absolute value squared)

$S_{V}=\left\langle\Delta V^{2}\right\rangle=\left|\lambda_{V}\right|^{2} 4 k_{B} T^{2} \frac{G_{\text {th }}^{\text {tot }}\left(G T G_{\text {th }}^{\text {tot }}-\alpha^{2}\right)+\omega^{2} C_{h} G T}{\alpha^{2}}$.

The term in parentheses is positive semidefinite due to the thermoelectric stability condition $\alpha^{2} \leq G T G_{\text {th }}^{\text {tot }}$ valid for all thermoelectric systems. The current-noise spectral density 
across the inductor, $S_{I}$, has the same form as $S_{V}$ in Eq. (6), but where $\lambda_{V}$ is replaced by $\lambda_{I}$.

The square of the NEP $P_{\text {ne }}^{2}$ is the power spectral density for which the induced-thermoelectric-voltage spectral density across the capacitor equals $S_{V}$, or for which the thermoelectric current spectral density across the inductor equals $S_{I}$. These yield the same results, $S_{V} /\left|\lambda_{V}\right|^{2}=$ $S_{I} /\left|\lambda_{I}\right|^{2}$,

$$
P_{\mathrm{ne}}^{2} \equiv \frac{S_{V}}{\left|\lambda_{V}\right|^{2}}=4 k_{B} T^{2} \frac{G_{\mathrm{th}}^{\mathrm{tot}}\left(G T G_{\mathrm{th}}^{\mathrm{tot}}-\alpha^{2}\right)+\omega^{2} C_{h}^{2} G T}{\alpha^{2}} .
$$

This may be written in a more tractable form by use of the zero-frequency thermoelectric figure of merit $z T=$ $\alpha^{2} /\left(G_{\text {th }}^{\text {tot }} G T-\alpha^{2}\right)[33]$ and the thermal time constant $\tau_{T}=$ $C_{h} / G_{\text {th }}^{\text {tot }}$ :

$$
P_{\mathrm{ne}}^{2}=\frac{4 k_{B} T^{2}\left[1+\omega^{2} \tau_{T}^{2}(1+z T)\right] G_{\mathrm{th}}^{\mathrm{tot}}}{z T} .
$$

The zero-frequency thermoelectric NEP thus equals the usual thermal bolometer NEP [34] from the thermal fluctuation noise divided by the square root of the figure of merit. Moreover, the thermal time constant determining the frequency band for the detection is increased by the factor $\sqrt{1+z T}$.

Above discussion disregards the contribution from the amplifier noise. This is discussed separately below.

The NEP written above can be optimized, as typically the overall level of the thermoelectric junction conductance can be chosen almost at will, and the coefficients $\alpha$ and $G_{\text {th }}$ scale with the same prefactor. In the sensor discussed here, this means optimizing the normal-state conductance of the thermoelectric junction. On the other hand, for a given absorber volume $\Omega$, the thermal conductance of the spurious process $G_{q \text {-ph }}$ cannot be affected much. Therefore, choosing a too small junction conductance results in a poor thermoelectric figure of merit $z T$, whereas increasing the junction conductance increases the thermal fluctuation noise and the Johnson-Nyquist current noise of the junction. In addition, a high junction conductance may lead to a heating of the normal-metal (ferromagnetic) electrode, thereby reducing the temperature gradient across the junction, and the associated thermoelectric effects. In what follows we assume that this electrode is thick enough so that its heating can be disregarded. For zerofrequency NEP, the optimum is obtained with conductance $G_{\mathrm{th}} / G_{\mathrm{ph}}=\sqrt{1+z T_{i}}$, where $z T_{i}=\alpha^{2} /\left(G_{\mathrm{th}} G T-\alpha^{2}\right)$ is the intrinsic figure of merit of the junction (not including the heat conductance to the phonons). With this choice, the optimal NEP of the thermoelectric detector is

$$
P_{\mathrm{ne}, \mathrm{opt}}^{2}=\frac{4 G_{q-\mathrm{ph}} k_{B} T^{2}\left(1+\sqrt{1+z T_{i}}\right)^{2}}{z T_{i}} .
$$

It hence reaches the limit set by the thermal fluctuation noise of the spurious process for $z T_{i} \rightarrow \infty$.

The above discussion holds for arbitrary thermoelectric sensors of the type depicted in Fig. 1(b). However, typically strong thermoelectric effects are found only above room temperature, which renders the thermal fluctuation noise very large. The combination of a spin-split superconductor with a spin-polarized contact circumvents this problem, leading to large thermoelectric response even at subkelvin temperatures. In the following, we analyze this system in more detail.

\section{SUPERCONDUCTOR-FERROMAGNET THERMOELECTRIC RADIATION DETECTOR}

First, following Ref. [19], we write the thermoelectric coefficients of the spin-polarized junction between the spin-split superconductor and the nonsuperconducting contact as

$$
\begin{gathered}
G=G_{T} \int_{-\infty}^{\infty} d E \frac{N_{0}(E)}{4 k_{B} T \cosh ^{2}\left(\frac{E}{2 k_{B} T}\right)}, \\
G_{\text {th }}=\frac{G_{T}}{e^{2}} \int_{-\infty}^{\infty} d E \frac{E^{2} N_{0}(E)}{4 k_{B} T^{2} \cosh ^{2}\left(\frac{E}{2 k_{B} T}\right)} \\
\alpha=\frac{G_{T}}{2 e} P \int_{-\infty}^{\infty} d E \frac{E N_{z}(E)}{4 k_{B} T \cosh ^{2}\left(\frac{E}{2 k_{B} T}\right)} .
\end{gathered}
$$

Here $G_{T}$ is the normal-state electrical conductance of the junction, and $N_{0}(E)=\left(N_{\uparrow}+N_{\downarrow}\right) / 2$ and $N_{z}=N_{\uparrow}-$ $N_{\downarrow}$ are the spin-averaged and the spin-difference density of states (DOS) of the superconductor, normalized to the normal-state DOS $v_{F}$ at the Fermi level. They are obtained from $N_{\uparrow / \downarrow}=N_{S}(E \mp h)$, with $N_{S}(E)=\operatorname{Re}[\mid E+$ $\left.i \Gamma \mid / \sqrt{(E+i \Gamma)^{2}-\Delta^{2}}\right]$, where $h$ is the spin-splitting field and $\Gamma \ll \Delta$ describes pair-breaking effects inside the superconductor. Analytic approximations for Eq. (10) are detailed in Ref. [19].

The heat capacity of the absorber with volume $\Omega$ is obtained from

$$
\begin{aligned}
C_{h} & =\frac{d}{d T}\left(v_{F} \Omega \int_{-\infty}^{\infty} d E E N_{0}(E) f_{\mathrm{eq}}(E)\right) \\
& =\frac{v_{F}}{4 k_{B} T^{2}} \int_{-\infty}^{\infty} \frac{E^{2} N_{0}(E)}{\cosh ^{2}\left(\frac{E}{2 k_{B} T}\right)}=\frac{v_{F} \Omega e^{2}}{G_{T}} G_{\mathrm{th}} .
\end{aligned}
$$

The thermal time constant of the junction, $C_{h} / G_{\text {th }}$, hence remains independent of superconductivity or spin splitting. 


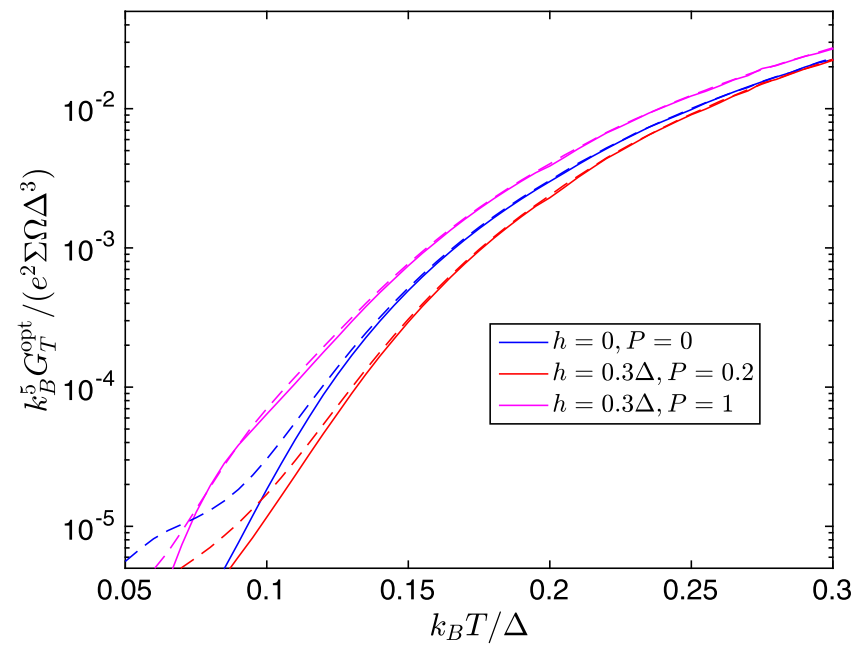

FIG. 2. Optimal normal-state tunnel conductance $G_{T}$ of the thermoelectric junction. For $\mathrm{Al}$ of volume $10^{-19} \mathrm{~m}^{3}$, a value of 1 corresponds to $G_{T}^{-1}=20 \Omega$. At the lowest temperatures the results depend strongly on the value of the broadening parameter $\Gamma$ used in the numerics. The solid lines are calculated with $\Gamma=10^{-4} \Delta$ and the dashed lines are calculated with $\Gamma=10^{-3} \Delta$.

The electron-phonon heat conductance of a spin-split superconductor in the pure limit can be obtained from [35]

$$
\begin{aligned}
G_{e-\mathrm{ph}}= & \frac{\Sigma \Omega}{96 \zeta(5) k_{B}^{5} T^{2}} \int_{-\infty}^{\infty} d E E \int_{-\infty}^{\infty} d \omega \\
& \times \omega^{2}|\omega| L_{E, E+\omega} F_{E, \omega},
\end{aligned}
$$

with

$$
F_{E, \omega}=-\frac{1}{2}\left[\sinh \left(\frac{\omega}{2 T}\right) \cosh \left(\frac{E}{2 T}\right) \cosh \left(\frac{E+\omega}{2 T}\right)\right]^{-1},
$$

$L_{E, E^{\prime}}=(1 / 2) \sum_{\sigma= \pm} N_{\sigma}(E) N_{\sigma}\left(E^{\prime}\right)\left\{1-\Delta^{2} /\left[(E+\sigma h)\left(E^{\prime}\right.\right.\right.$ $+\sigma h)]\}$, and $\sigma= \pm$ for spin $\uparrow / \downarrow$. Here $\Sigma$ is the materialdependent electron-phonon coupling constant (for typical values, see Ref. [34]), $\Omega=$ wld is the volume of the superconductor island, and $\zeta(x)$ is the Riemann zeta function.

In the low-temperature limit $k_{B} T \ll \Delta-|h|$, the electron-phonon heat conductance can be approximated as

$$
\begin{aligned}
G_{e-\mathrm{ph}}= & \frac{\Sigma \Omega}{96 \zeta(5)} T^{4}\left[\cosh \tilde{h} e^{-\tilde{\Delta}} f_{1}(\tilde{\Delta})\right. \\
& \left.+\pi \tilde{\Delta}^{5} e^{-2 \tilde{\Delta}} f_{2}(\tilde{\Delta})\right],
\end{aligned}
$$

where $\tilde{h}=h / k_{B} T$ and $\tilde{\Delta}=\Delta / k_{B} T$. The function $f_{1}$ can be approximated with an expansion $f_{1}(\tilde{\Delta})=\sum_{n=1}^{\infty} C_{n} / \tilde{\Delta}^{n}$ with coefficients $C_{0} \approx 440, C_{1} \approx-500, C_{2} \approx 1400$, and $C_{3} \approx-4700$. An expansion for $f_{2}$ is $f_{2}(\tilde{\Delta})=\sum_{n=1}^{\infty} B_{n} / \tilde{\Delta}^{n}$ with coefficients $B_{0}=64, B_{0}=64, B_{1}=144$, and $B_{2}=$ 258. The derivation of Eq. (14) is presented in the Appendix.

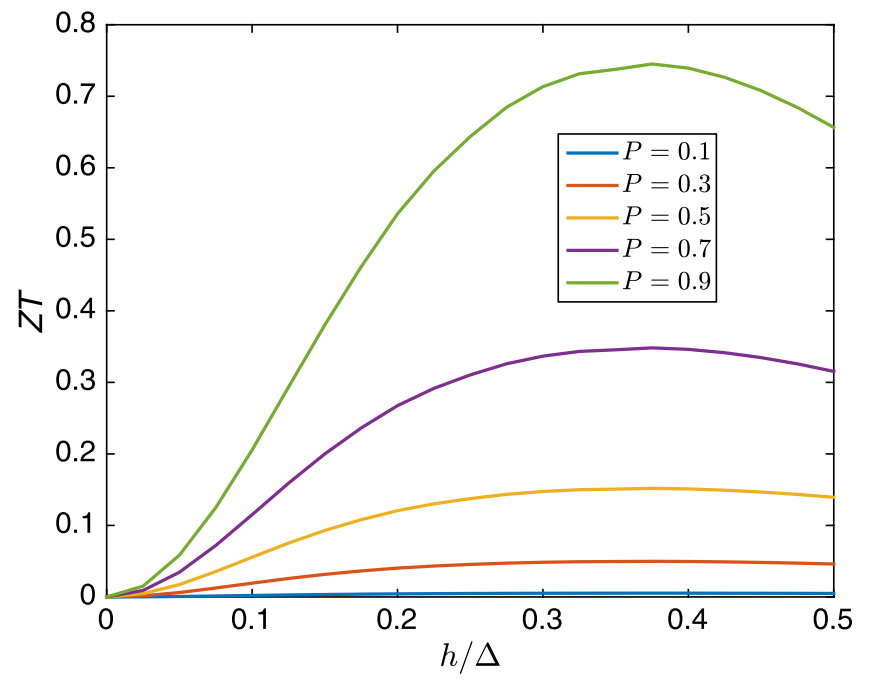

FIG. 3. Thermoelectric figure of merit as a function of the exchange field for junctions with different polarizations $P$ at temperature $T=0.1 \Delta / k_{B}$, with $G_{T}=5 \times 10^{-4} e^{2} \Sigma \Omega \Delta^{3}$ and $\Gamma=10^{-3} \Delta$.

In the following, we use the above formulas to discuss the behavior of the SFTED. For this, we evaluate the above integrals numerically to obtain predictions of the optimal junction conductance, the thermoelectric figure of merit $z T$, the total NEP, and the time constant. The optimal normal-state junction conductance $G_{T}$ is plotted as a function of temperature in Fig. 2. As the electron-phonon heat conductance dies out faster than the junction heat conductance at low temperatures, the optimal $G_{T}$ also depends strongly on temperature. Note that for an $\mathrm{Al}$ absorber with electron-phonon coupling constant $\Sigma=0.3 \times 10^{9} \mathrm{~W} / \mathrm{m}^{3} \mathrm{~K}^{5}$, volume $\Omega=$ $10^{-19} \mathrm{~m}^{3}$, and $\Delta=200 \mu \mathrm{eV}$, the dimensionless parameter $k_{B}^{5} G_{T} /\left(e^{2} \Sigma \Omega \Delta^{3}\right)=G_{T} \times 20 \Omega$. The optimal normal-state resistance of the junction is thus within the range from $20 \mathrm{k} \Omega$ to $20 \mathrm{M} \Omega$. Moreover, since both $G_{T}$ and $\Sigma \Omega$ depend on the area of the absorber, the real optimizable parameters are the absorber film thickness and the junction conductance per unit area. In what follows, we use $G_{T}=5 \times 10^{-4} e^{2} \Sigma \Omega \Delta^{3}$, corresponding to a junction resistance of $40 \mathrm{k} \Omega$, optimal roughly at $T \approx 0.1 \Delta / k_{B} \sim$ $200 \mathrm{mK}$. This corresponds to a resistance times unit area of $400 \mathrm{k} \Omega \mu \mathrm{m}^{2}$, which is quite easily reached with $\mathrm{AlO}_{2}$ tunnel junctions [36], but would be somewhat challenging for spin-filter EuS barriers, ranging typically between 10 and $1000 \mathrm{M} \Omega \mu \mathrm{m}^{2}$ [25].

On the other hand, the thermoelectric figure of merit $z T$ depends strongly on the detector polarization. We show this by plotting $z T$ for the parameters indicated above as a function of the exchange field at $T=0.1 \Delta / k_{B}$ in Fig. 3 . Because of the presence of the electron-phonon process acting as an extra heat channel, the figure of merit does not exceed unity. 


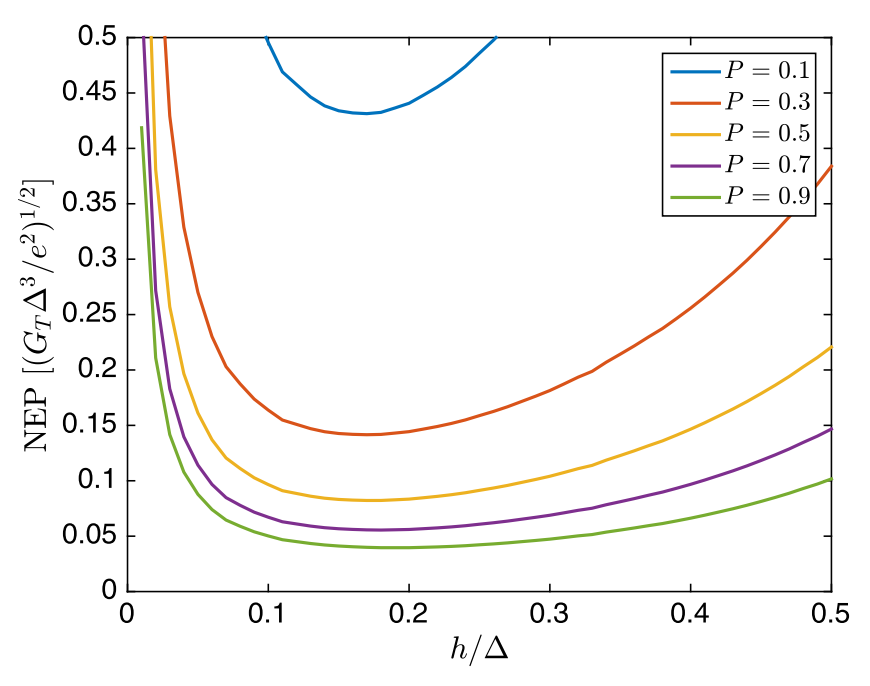

FIG. 4. Zero-frequency NEP as a function of the exchange field for junctions with different polarizations $P$ at temperature $T=0.1 \Delta / k_{B}$, with $G_{T}=5 \times 10^{-4} e^{2} \Sigma \Omega \Delta^{3}$ and $\Gamma=10^{-3} \Delta$. For the parameters considered in this paper, $\sqrt{G_{T} \Delta^{3} / e^{2}} \approx$ $10^{-18} \mathrm{~W} / \sqrt{\mathrm{Hz}}$.

The most interesting characteristic of any detector is its sensitivity, in this case the NEP. We plot this as a function of exchange field in Fig. 4 and as a function of temperature in Fig. 5. The NEP is normalized to $\sqrt{G_{T} \Delta^{3} / e^{2}}$, which corresponds to approximately $10^{-18} \mathrm{~W} / \sqrt{\mathrm{Hz}}$ for the parameters chosen. The dashed line in Fig. 5 indicates the $\mathrm{NEP}_{\text {bolometer }}=4 G_{q \text {-ph }} T^{2}$ obtained for a TES with the same absorber volume at the corresponding temperature, with its heat conductance limited by electron-phonon coupling (i.e., a hot-electron TES [37]). In Fig. 4 that reference value is exactly unity for the parameters chosen. As the TES operates in the dissipative regime at the transition, the normal-state value for $G_{q \text {-ph }}$ must be used. Moreover, this estimate disregards the bias-induced heating, which sets the operating temperature higher than the bath temperature, and extra noise sources often found in TES realizations. We find that a SFTED can reach similar or better values than such a TES even with quite modest values of the junction polarization at low temperatures. Note that these results depend a bit on the precise value of the junction conductance - with a higher conductance, NEP at higher temperatures would be lower (see Fig. 2), and vice versa.

In practice, the most-sensitive TES bolometers to date have been fabricated from suspended structures where the thermal conductance to the bath is limited by phonon transport, achieving NEP on the order of $1 \times 10^{-19} \mathrm{~W} / \sqrt{\mathrm{Hz}}$ $[38,39]$ at $T_{c}$ of around $100 \mathrm{mK}$. On the basis of Fig. 5, the SFTED is also predicted to reach a lower NEP than that.

For completeness, we show the behavior of the thermal time constant $\tau^{*}=\tau_{T} \sqrt{1+z T}$ as a function of temperature in Fig. 6. It is given in the units of $\tau_{0}=v_{F} \Omega e^{2} / G_{T}$.

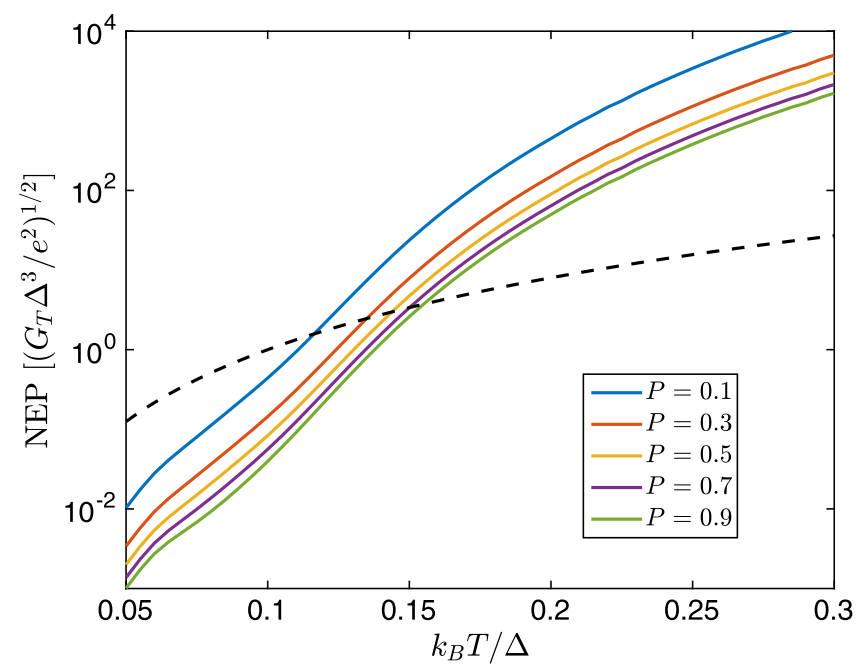

FIG. 5. Zero-frequency NEP as a function of the temperature for junctions with different polarizations $P$ with exchange field $h=0.2 \Delta$, with $G_{T}=5 \times 10^{-4} e^{2} \Sigma \Omega \Delta^{3}$ and $\Gamma=10^{-3} \Delta$. For the parameters considered in this paper, $\sqrt{G_{T} \Delta^{3} / e^{2}} \approx$ $10^{-18} \mathrm{~W} / \sqrt{\mathrm{Hz}}$. The dashed line shows the thermal-fluctuationnoise NEP $=\sqrt{20 \Sigma \Omega T^{6}}$ for a TES of the same volume.

For $v_{F}=10^{47} \mathrm{~J}^{-1} \mathrm{~m}^{-3}, \Omega=10^{-19} \mathrm{~m}^{3}$, and $G_{T}^{-1}=2 \mathrm{M} \Omega$, $\tau_{0} \approx 0.1 \mathrm{~ms}$. At low temperatures, the tunnel junction dominates the heat conductance, and $\tau^{*} \approx \tau_{0}$. In this case $z T$ is also appreciable, and slightly modifies $\tau^{*}$. On the other hand, at high temperatures electron-phonon heat conduction takes over, and the detector becomes faster. To illustrate this crossover, we show the time constant for two different values of $G_{T}$.

The above results are obtained by disregarding spin relaxation. Aluminum is a light material, and therefore the spin-orbit scattering in it is typically quite weak, and the spin relaxation is dominated by spin-flip scattering. The typical spin relaxation times $\tau_{s r}$ in $\mathrm{Al}$ are on the order of $100 \mathrm{ps}$ [40], and therefore $\hbar /\left(\tau_{s r} \Delta\right) \sim 0.03$, and the model disregarding spin relaxation is more or less justified. However, spin-flip scattering in the presence of exchange field yields a nonzero DOS inside the superconducting gap, and eventually leads to pair breaking [22]. Above, such effects are taken into account with the parameter $\Gamma$. For heavier materials, such as $\mathrm{Nb}$, spin relaxation is caused by spin-orbit scattering, and the thermoelectric effects become weaker. Therefore, use of such heavier materials, for example, to increase the operation temperature of the thermoelectric detector beyond the critical temperature of $\mathrm{Al}$, would require further analysis of the effects of spin relaxation.

\section{A. Contribution of amplifier noise}

In the preceding analysis we disregard the noise due to the voltage or current measurement. We can include it 


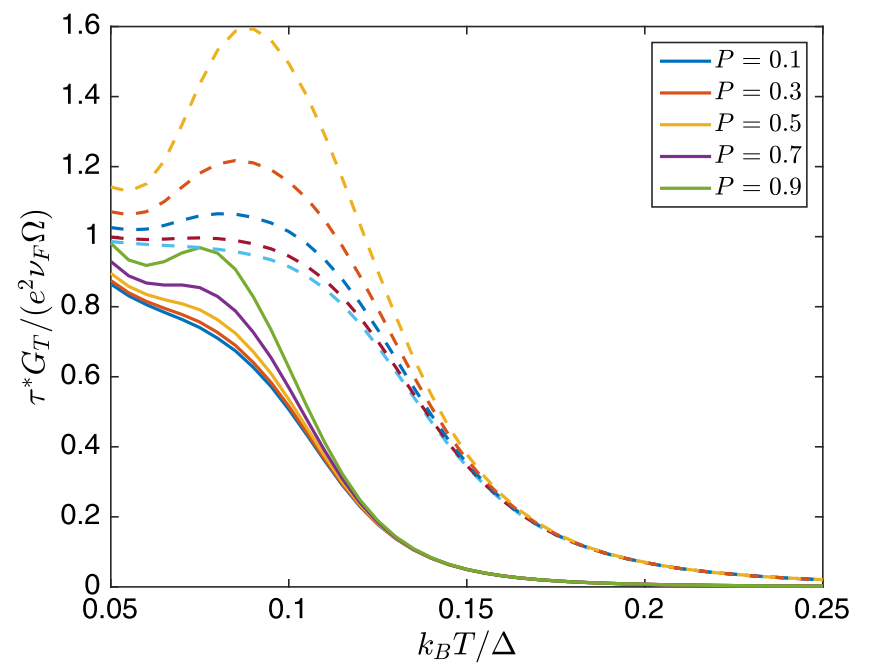

FIG. 6. Temperature dependence of the detector time constant $\tau^{*}$ determining the (angular) frequency bandwidth $1 / \tau^{*}$ where the NEP is low. We chose $h=0.2 \Delta$. The solid lines are calculated with $G_{T}=5 \times 10^{-4} e^{2} \Sigma \Omega \Delta^{3}$, whereas the dashed lines correspond to 10 times larger conductance. For the former, $\tau_{0} \approx$ $0.1 \mathrm{~ms}$, and for the latter, $\tau_{0}=0.01 \mathrm{~ms}$.

by assuming an added voltage-noise spectral density $S_{V}^{A}$ or current-noise spectral density $S_{I}^{A}$ for the amplifier used for voltage or current measurement. In the case of voltage measurements, the amplifier adds to NEP the contribution (for $\omega=0$, for simplicity)

$$
P_{\mathrm{ne}, A, V}^{2}=\frac{S_{V}^{A} G T G_{\mathrm{th}}^{\mathrm{tot}}}{z T(1+z T)}
$$

whereas in the case of current measurement the contribution is

$$
P_{\mathrm{ne}, A, I}^{2}=\frac{S_{I}^{A}(1+z T) G_{\mathrm{th}}^{\mathrm{tot}} T}{G z T} .
$$

We can hence see that the relative contribution from the voltage amplifier to the overall NEP decreases as the thermoelectric junction resistance increases. On the other hand, in the case of current measurement, the amplifier contribution becomes independent of the junction resistance when $G_{\text {th }}^{\text {tot }}$ is dominated by the junction heat conductance. Another way to estimate the contribution of amplifier noise is by dividing the corresponding NEPs by the total thermoelectric NEP from Eq. (8) (at $\omega=0)$. We hence get

$$
\begin{gathered}
r_{V} \equiv \frac{P_{\mathrm{ne}, A, V}^{2}}{P_{\mathrm{ne}}^{2}}=\frac{S_{V}^{A}}{4 k_{B} T} \frac{G}{(1+z T)}, \\
r_{I} \equiv \frac{P_{\mathrm{ne}_{A}, I}^{2}}{P_{\mathrm{ne}}^{2}}=\frac{S_{I}^{A}}{4 k_{B} T} \frac{(1+z T)}{G} .
\end{gathered}
$$

A typical good voltage preamplifier for low-frequency measurements has a voltage noise on the order of $\sqrt{S_{V}}=1.5 \mathrm{nV} / \sqrt{\mathrm{Hz}}$ at room temperature and $\sqrt{S_{V}}=$ $0.3 \mathrm{nV} / \sqrt{\mathrm{Hz}}$ at cryogenic temperatures [41]. Together with the normal-state tunnel conductance used above, and the $\Delta$ for $\mathrm{Al}$, the latter value yields the relative NEP for voltage measurements as $r_{V} \approx G \Delta /\left(G_{T} k_{B} T\right)(1+z T)^{-1}$. This is much below unity in the entire relevant temperature range $\left(k_{B} T \ll \Delta\right)$ due to the exponential suppression of $G$. On the other hand, a very good current amplifier can have an added noise of $\sqrt{S_{I}}=0.5 \mathrm{fA} / \sqrt{\mathrm{Hz}}$. With that value we get $r_{I} \approx 10^{-4} \Delta /\left(k_{B} T\right) \times(1+z T) G_{T} / G$. This exceeds unity below $k_{B} T \approx 0.1 \Delta$ (the precise value depends on the exchange field chosen), and the current measurement accuracy starts limiting the thermoelectric detector NEP for $T \lesssim 0.1 \Delta / k_{B}$. This difference between the two types of measurements originates from the fact that the thermoelectric voltage can be on the order of the temperature difference itself due to the thermopower on the order of $k_{B} / e$, whereas the thermoelectric current is exponentially suppressed [19] and hence harder to measure. However, ultimately at very low temperatures the voltage measurement also becomes harder as it requires the voltmeter impedance to far exceed that of the junction, and this condition becomes harder to meet at low temperatures.

Typical absolute-thermometer-based radiation detectors operating at the bath temperature (i.e., in contrast to, for example, a TES, where the bias sets the operating point above bath temperature) suffer from chip temperature fluctuations due to fluctuations in the cooling power. However, a thermoelectric detector measures a temperature difference $\Delta T$ instead of the absolute temperature. Because the chip temperature fluctuations affect both the temperature of the absorber and that of the measurement electrode, they do not affect $\Delta T$ (to the lowest order). This is an added benefit for TEDs in comparison with detectors based on resistance or inductance measurements.

\section{CONCLUSIONS}

Thermoelectric radiation detection is not a new concept [15]. However, most of the previously studied thermoelectric detectors have relied on using semiconducting thermoelectric materials, operating at and above room temperature $T_{\mathrm{RT}}$. Because the spurious heat-conduction processes have heat conductance that scales at least as approximately $T^{3}$ (typical phonon heat conductivity [34]), the corresponding NEP is $\left(T_{\mathrm{RT}} / T\right)^{5 / 2} \sim 10^{15 / 2}$ times larger than that considered here (this estimate assumes the Debye temperature exceeds room temperature, but it should in any case be taken as indicative). On the other hand, quantum-dot structures may exhibit strong thermoelectric effects even at low temperatures [42]. In contrast to the superconductor-ferromagnet structure considered here, in those devices the thermoelectric effects are single-channel 


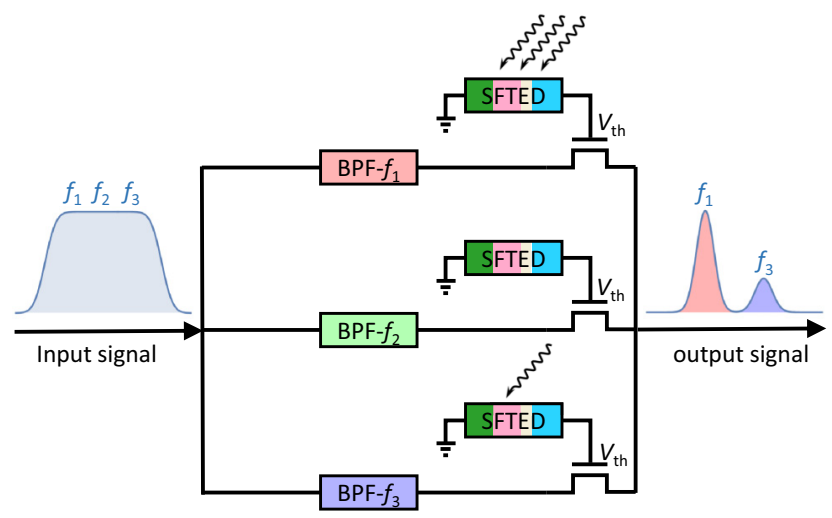

FIG. 7. One possible scheme for frequency-domain multiplexing of the thermoelectric detectors. Here a broadband electromagnetic wave, or a frequency comb, is divided into different frequency components via narrow-band-pass filters (BPFs), and directed through field-effect transistors whose conductance is modulated by the voltage from the detectors. As a result, the output spectrum contains pixel-specific information about the absorbed radiation power.

phenomena, and therefore it may be difficult to make the electronic thermal conduction dominate over the spurious heat-conduction channels.

In this paper, we show how a combination of superconducting and magnetic materials can be used to construct a low-temperature radiation detector relying on the thermoelectric effect and thereby not requiring extra bias power to be applied to the device. The proposed device thus differs qualitatively from the other previously known superconducting detectors such as TES and KID. The lack of the need to have extra bias power leads to simpler designs of arrays of such detectors, and helps in maintaining the low operating temperatures required for ultrasensitive operation. In addition, ultrasensitive TES bolometers necessarily have a very low tolerance for excess power loading, as the device can be saturated and pushed out of the transition region with it. For the detectors discussed here, there is no such abrupt effect, although excess power could lead to performance degradation due to overheating. Nevertheless, because of the lack of the bias lines, novel multiplexing strategies may need to be designed. We present one possible scheme in Fig. 7. There the output looks quite similar to that of frequency-multiplexed TES or KID read-out schemes, but the possible heating effects in the (dissipative) field-effect transistors can be engineered far apart from the pixels absorbing the radiation. Nevertheless, the optimal multiplexing strategies are a topic for further research.

\section{ACKNOWLEDGMENTS}

This project was supported by the Academy of Finland via its Key Funding Project (Grant No. 305256), the Center of Excellence program (Grant No. 284594), and Grants No. 298667 and 317118; the European Union Seventh Framework Programme (ERC Grant No. 615187COMANCHE) and the Horizon 2020 research and innovation programme (Grant No. 800923-SUPERTED); the Tuscany Region under the FARFAS 2014 project SCIADRO, and the Spanish Ministerio de Economia, Industria y Competitividad (Grants No. FIS2014-55987- P and No. FIS2017-82804-P).

\section{APPENDIX: ELECTRON-PHONON HEAT CONDUCTANCE}

We calculate the electron-phonon heat conductance coefficient $G_{e \text {-ph }}$ beginning from Eq. (12). We first extract the temperature-dependent prefactor by scaling all the quantities with dimensions of energy by temperature; for example, $\tilde{E}=E / k_{B} T$. The scaled quantities are dimensionless and are denoted with a tilde over the variable. We then change the integration variables to $x=\tilde{E}$ and $y=\tilde{E}+\tilde{\omega}$. We obtain

$$
G_{e-\mathrm{ph}}=\frac{\Sigma \Omega T^{4}}{96 \zeta(5)} \sum_{\sigma= \pm} \frac{I^{\sigma}}{4},
$$

where

$$
\begin{aligned}
I^{\sigma}= & \iint d x d y \operatorname{sgn}[(x+\sigma \tilde{h})(y+\sigma \tilde{h})] \\
& \times \frac{x|x-y|^{3}\left[(x+\sigma \tilde{h})(y+\sigma \tilde{h})-\tilde{\Delta}^{2}\right]}{\sqrt{\left([x+\sigma \tilde{h}]^{2}-\tilde{\Delta}^{2}\right)\left([y+\sigma \tilde{h}]^{2}-\tilde{\Delta}^{2}\right)}} \frac{4 e^{-(|x|+|y|) / 2}}{\sinh \frac{x-y}{2}} .
\end{aligned}
$$

Above, both $x$ and $y$ are integrated from $-\infty$ to $+\infty$, excluding the region $[-\tilde{\Delta}-\sigma \tilde{h}, \tilde{\Delta}-\sigma \tilde{h}]$, in which the spin-split DOS vanishes. We also assume that $\Delta-h \gg$ $k_{B} T$ so that we can make an approximation

$$
\cosh x \approx \frac{e^{|x|}}{2}, \quad x>\tilde{\Delta}-\tilde{h},
$$

and similarly for $\cosh y$.

The integral is divided into four separate quadrants by the gaps in the DOS. The integral over the quadrant $n$ for the spin $\sigma$ is $I_{n}^{\sigma}$. Because the integrand of Eq. (A2) is symmetric with respect to simultaneous inversion of $x, y$, and $\sigma$, the contributions from the opposing quadrants are equal:

$$
I=\frac{1}{4} \sum_{n=1}^{4} \sum_{\sigma= \pm} I_{n}^{\sigma}=\frac{1}{2} \sum_{\sigma= \pm}\left(I_{1}^{\sigma}+I_{2}^{\sigma}\right) .
$$

We calculate the integral over the first quadrant. This part of the integral represents scattering processes, for which 
$x, y>0$. Physically, this means that, the interacting quasiparticles are both particlelike. By shifting the integration limits, we get

$$
\begin{aligned}
I_{1}^{\sigma}= & \int_{\tilde{\Delta}-\sigma \tilde{h}}^{\infty} d x \int_{\tilde{\Delta}-\sigma \tilde{h}}^{\infty} d y \\
& \times \frac{x|x-y|^{3}\left[(x+\sigma h)(y+\sigma h)-\tilde{\Delta}^{2}\right]}{\sqrt{\left[(x+\sigma h)^{2}-\tilde{\Delta}^{2}\right]\left[(y+\sigma h)^{2}-\tilde{\Delta}^{2}\right]}} \frac{8}{e^{x}-e^{y}} \\
= & \int_{0}^{\infty} d x \int_{0}^{\infty} d y \\
& \times \frac{(x+\tilde{\Delta}-\sigma \tilde{h})|x-y|^{3}[x y+(x+y) \tilde{\Delta}]}{\left(e^{x}-e^{y}\right) \sqrt{x y(x+2 \tilde{\Delta})(y+2 \tilde{\Delta})}} 8 e^{-\tilde{\Delta}+\sigma \tilde{h}} .
\end{aligned}
$$

Above, we have $\tilde{h}$ dependence in two places: in the exponential outside the integral and as a linear term in the numerator. However, the parts of the numerator that are symmetric with respect to exchange $x \leftrightarrow y$ do not contribute to the integral. Therefore, we can write the integral as

$$
\begin{aligned}
I_{1}^{\sigma}= & e^{-\tilde{\Delta}+\sigma \tilde{h}} \int_{0}^{\infty} d x \int_{0}^{\infty} d y \\
& \times \frac{8 x^{2}|x-y|^{3}(y+\tilde{\Delta})}{\left(e^{x}-e^{y}\right) \sqrt{x y(x+2 \tilde{\Delta})(y+2 \tilde{\Delta})}} \\
= & e^{-\tilde{\Delta}+\sigma \tilde{h}} f_{1}(\tilde{\Delta}),
\end{aligned}
$$

where $f_{1}(\tilde{\Delta})$ is a monotonically increasing function with values $f(2) \approx 326$ and $\lim _{\tilde{\Delta} \rightarrow \infty} f_{1}(\tilde{\Delta}) \approx 438$. We can perform a Taylor expansion $f_{1}(\tilde{\Delta})=\sum_{n=0}^{\infty} C_{n} / \tilde{\Delta}^{n}$ by first expanding the integrand into series in $\tilde{\Delta}^{-1}$ and then integrating separately for each term. The first few coefficients are $C_{0} \approx 440, C_{1} \approx-500, C_{2} \approx 1400$, and $C_{3} \approx-4700$.

Doing the sum over the spins, we find the contribution from the first quadrant:

$$
I_{1}=\sum_{\sigma= \pm} I_{1}^{\sigma}=2 \cosh \tilde{h} e^{-\tilde{\Delta}} f_{1}(\tilde{\Delta})
$$

The second quadrant describes the contribution from the recombination processes, for which one quasiparticle is holelike $(x<0)$ and the other is particlelike $(y>0)$ :

$I_{2}^{\sigma}=-\int_{-\infty}^{-\tilde{\Delta}-\sigma \tilde{h}} d x \int_{\tilde{\Delta}-\sigma \tilde{h}}^{\infty} d y \frac{8 e^{x}}{e^{x}-e^{y}}$

$$
\begin{aligned}
& \times \frac{x(x-y)^{3}\left[(x+\sigma h)(y+\sigma h)-\tilde{\Delta}^{2}\right]}{\sqrt{\left[(x+\sigma h)^{2}-\tilde{\Delta}^{2}\right]\left[(y+\sigma h)^{2}-\tilde{\Delta}^{2}\right]}} \\
& =\int_{0}^{\infty} d x \int_{0}^{\infty} d y \frac{(x+y+2 \tilde{\Delta})^{3}\left[x y+(x+y) \tilde{\Delta}+2 \tilde{\Delta}^{2}\right]}{\sqrt{x y(2 \tilde{\Delta}+x)(2 \tilde{\Delta}+y)}} \\
& \times 8(x+\tilde{\Delta}+\sigma \tilde{h}) e^{-x-y} e^{-2 \tilde{\Delta}},
\end{aligned}
$$

where we approximate $e^{2 \Delta+x+y}-1 \approx e^{2 \Delta+x+y}$. Above, the exchange field $\sigma \tilde{h}$ appears only as a linear term. If we sum over the two spin directions, terms odd in $\sigma$ cancel and we can write $I_{2}$ in the form

$$
I_{2}=\sum_{\sigma} I_{2}^{\sigma}=2 \pi \tilde{\Delta}^{5} e^{-2 \tilde{\Delta}} f_{2}(\tilde{\Delta}) .
$$

Within the approximation given by Eq. (A3), the exchange field does not modify the contribution from the recombination processes.

The function $f_{2}$ is defined as

$$
\begin{aligned}
f_{2}(\tilde{\Delta})= & \int_{0}^{\infty} d x \int_{0}^{\infty} d y \\
& \times \frac{(x+y+2 \tilde{\Delta})^{3}\left[x y+(x+y) \tilde{\Delta}+2 \tilde{\Delta}^{2}\right]}{\pi \tilde{\Delta}^{5} \sqrt{x y(2 \tilde{\Delta}+x)(2 \tilde{\Delta}+y)}} \\
& \times 8 e^{-x-y}(x+\tilde{\Delta}) .
\end{aligned}
$$

The function $f_{2}$ is a monotonically decreasing function with values $f_{2}(4) \approx 123$ and $\lim _{\tilde{\Delta} \rightarrow \infty} f_{2}(\tilde{\Delta})=64$. An expansion $f_{2}(\tilde{\Delta})=\sum_{n=0}^{\infty} B_{n} / \tilde{\Delta}^{n}$ is obtained by first expanding the integrand asymptotically at $\tilde{\Delta}=\infty$ and then calculating the integral term by term. The first few coefficients are $B_{0}=64, B_{1}=144, B_{2}=258$, and $B_{3}=693 / 2$.

By combining Eqs. (A1), (A4), (A7), and (A9), we find the electron-phonon heat conductance for a spin-split superconductor: Eq. (14). At low temperatures, when $\tilde{\Delta} \gg$ 1 , scattering processes dominate the heat conductance. The two processes become of the same order of magnitude when $k_{B} T \approx 0.1 \Delta$. At high temperatures, recombination processes dominate.

[1] D. Hanson, S. Hoover, A. Crites, P. Ade, K. Aird, J. Austermann, J. Beall, A. Bender, B. Benson, and L. Bleem et al., Detection of B-Mode Polarization in the Cosmic Microwave Background with Data from the South Pole Telescope, Phys. Rev. Lett. 111, 141301 (2013).

[2] P. Ade, Y. Akiba, A. Anthony, K. Arnold, M. Atlas, D. Barron, D. Boettger, J. Borrill, S. Chapman, and Y. Chinone 
et al., A measurement of the cosmic microwave background b-mode polarization power spectrum at subdegree scales with polarbear, Astrophys. J. 794, 171 (2014).

[3] M. Madhavacheril, N. Sehgal, R. Allison, N. Battaglia, J. R. Bond, E. Calabrese, J. Caligiuri, K. Coughlin, D. Crichton, and R. Datta et al., Evidence of Lensing of the Cosmic Microwave Background by Dark Matter Halos, Phys. Rev. Lett. 114, 151302 (2015).

[4] D. Farrah, K. E. Smith, D. Ardila, C. M. Bradford, M. Dipirro, C. Ferkinhoff, J. Glenn, P. Goldsmith, D. Leisawitz, and T. Nikola et al., Far-infrared instrumentation and technology development for the next decade, arXiv: 1709.02389.

[5] M. K. A. Luukanen, R. Appleby, and N. Salmon, in Millimeter-Wave and Terahertz Imaging in Security Applications, Terahertz Spectroscopy and Imaging, edited by K. E. Peiponen, A. Zeitler and M. Kuwata-Gonokami (Springer, Berlin Heidelberg, 2013), Chap., p. 491.

[6] J. N. Ullom and D. A. Bennett, Review of superconducting transition-edge sensors for x-ray and gammaray spectroscopy, Supercond. Sci. Technol. 28, 084003 (2015).

[7] M. Palosaari, M. Käyhkö, K. Kinnunen, M. Laitinen, J. Julin, J. Malm, T. Sajavaara, W. Doriese, J. Fowler, and C. Reintsema et al., Broadband Ultrahigh-Resolution Spectroscopy of Particle-Induced X Rays: Extending the Limits of Nondestructive Analysis, Phys. Rev. Appl. 6, 024002 (2016).

[8] L. Miaja-Avila, G. C. O’Neil, Y. I. Joe, B. K. Alpert, N. H. Damrauer, W. B. Doriese, S. M. Fatur, J. W. Fowler, G. C. Hilton, and R. Jimenez et al., Ultrafast Time-Resolved Hard X-Ray Emission Spectroscopy on a Tabletop, Phys. Rev. X 6, 031047 (2016).

[9] E. N. Grossman, D. G. McDonald, and J. Sauvageau, Farinfrared kinetic-inductance detectors, IEEE Trans. Magn. 27, 2677 (1991).

[10] N. Bluzer and M. G. Forrester, Superconducting quantum detectors, Opt. Eng. 33, 697 (1994).

[11] A. Sergeev, V. Mitin, and B. Karasik, Ultrasensitive hotelectron kinetic-inductance detectors operating well below the superconducting transition, Appl. Phys. Lett. 80, 817 (2002).

[12] F. Giazotto, T. T. Heikkilä, G. P. Pepe, P. Helistö, A. Luukanen, and J. P. Pekola, Ultrasensitive proximity josephson sensor with kinetic inductance readout, Appl. Phys. Lett. 92, 162507 (2008).

[13] J. Govenius, R. Lake, K. Tan, and M. Möttönen, Detection of Zeptojoule Microwave Pulses Using Electrothermal Feedback in Proximity-Induced Josephson Junctions, Phys. Rev. Lett. 117, 030802 (2016).

[14] P. K. Day, H. G. LeDuc, B. A. Mazin, A. Vayonakis, and J. Zmuidzinas, A broadband superconducting detector suitable for use in large arrays, Nature 425, 817 (2003).

[15] R. C. Jones, The ultimate sensitivity of radiation detectors, JOSA 37, 879 (1947).

[16] A. Varpula, A. V. Timofeev, A. Shchepetov, K. Grigoras, J. Hassel, J. Ahopelto, M. Ylilammi, and M. Prunnila, Thermoelectric thermal detectors based on ultra-thin heavily doped single-crystal silicon membranes, Appl. Phys. Lett. 110, 262101 (2017).

[17] D. V. Vechten, K. Wood, G. Fritz, A. Gyulamiryan, V. Nikogosoyan, N. Giordano, T. Jacobs, and A. Gulian,
Thermoelectric single-photon detectors: Isotropic seebeck sensors, in Eighteenth International Conference on Thermo- electrics, IEEE, Baltimore, (1999).

[18] Patent pending, filing number IT 102017000107007.

[19] A. Ozaeta, P. Virtanen, F. Bergeret, and T. Heikkilä, Predicted Very Large Thermoelectric Effect in FerromagnetSuperconductor Junctions in the Presence of a SpinSplitting Magnetic Field, Phys. Rev. Lett. 112, 057001 (2014).

[20] P. Machon, M. Eschrig, and W. Belzig, Nonlocal Thermoelectric Effects and Nonlocal Onsager Relations in a ThreeTerminal Proximity-Coupled Superconductor-Ferromagnet Device, Phys. Rev. Lett. 110, 047002 (2013).

[21] S. Kolenda, M. J. Wolf, and D. Beckmann, Observation of Thermoelectric Currents in High-Field SuperconductorFerromagnet Tunnel Junctions, Phys. Rev. Lett. 116, 097001 (2016).

[22] F. S. Bergeret, M. Silaev, P. Virtanen, and T. T. Heikkila, Nonequilibrium effects in superconductors with a spinsplitting field, arXiv:1706.08245.

[23] F. Giazotto, P. Solinas, A. Braggio, and F. Bergeret, Ferromagnetic-Insulator-Based Superconducting Junctions as Sensitive Electron Thermometers, Phys. Rev. Appl. 4, 044016 (2015).

[24] The system presented in Fig. 1 is perhaps the simplest realization that does not require an external magnetic field. Alternatively, the upper superconductorinsulator-ferromagnet part could be replaced by either superconductor-ferromagnetic insulator-normal metal or superconductor-ferromagnetic insulator-superconductor, where the ferromagnetic insulator would act as the spin filter. In that case it would also be possible to remove the lower ferromagnetic insulator

[25] J. Moodera, X. Hao, G. Gibson, and R. Meservey, ElectronSpin Polarization in Tunnel Junctions in Zero Applied Field with Ferromagnetic Eus Barriers, Phys. Rev. Lett. 61, 637 (1988).

[26] P. Tedrow, J. Tkaczyk, and A. Kumar, Spin-Polarized Electron Tunneling Study of an Artificially Layered Superconductor with Internal Magnetic Field: Euo-al, Phys. Rev. Lett. 56, 1746 (1986).

[27] A. M. Clogston, Upper Limit for the Critical Field in Hard Superconductors, Phys. Rev. Lett. 9, 266 (1962).

[28] J. Alexander, T. Orlando, D. Rainer, and P. Tedrow, Theory of fermi-liquid effects in high-field tunneling, Phys. Rev. B 31, 5811 (1985).

[29] R. Meservey and P. Tedrow, Spin-polarized electron tunneling, Phys. Rep. 238, 173 (1994).

[30] J. S. Moodera and R. H. Meservey, in Magnetoelectronics, edited by M. Johnson (Academic Press, San Diego, 2004), p. 151.

[31] J. S. Moodera, T. S. Santos, and T. Nagahama, The phenomena of spin-filter tunnelling, J. Phys. Condens. Matter 19, 165202 (2007).

[32] We choose the convention where the heat currents are into the absorber, and charge currents are out of it. Alternative conventions lead to some sign changes in the equations.

[33] Note that $z T$ is not the zero-frequency limit of $Z T$, but should be considered as a definition.

[34] F. Giazotto, T. T. Heikkilä, A. Luukanen, A. M. Savin, and J. P. Pekola, Opportunities for mesoscopics in thermometry 
and refrigeration: Physics and applications, Rev. Mod. Phys. 78, 217 (2006).

[35] P. Virtanen, T. Heikkilä, and F. Bergeret, Stimulated quasiparticles in spin-split superconductors, Phys. Rev. B 93, 014512 (2016).

[36] E. Strambini, V. Golovach, G. De Simoni, J. Moodera, F. Bergeret, and F. Giazotto, Revealing the magnetic proximity effect in eus/al bilayers through superconducting tunneling spectroscopy, Phys. Rev. Mater. 1, 054402 (2017).

[37] B. S. Karasik and R. Cantor, Demonstration of high optical sensitivity in far-infrared hot-electron bolometer, Appl. Phys. Lett. 98, 193503 (2011).

[38] A. D. Beyer, M. Kenyon, P. Echternach, B. Bumble, M. Runyan, T. Chui, C. Bradford, W. Holmes, and J. Bock, in Millimeter, Submillimeter, and Far-Infrared
Detectors and Instrumentation for Astronomy VI (International Society for Optics and Photonics, 2012), Vol. 8452, p. $84520 \mathrm{G}$.

[39] T. Suzuki, P. Khosropanah, M. Ridder, R. Hijmering, J. Gao, H. Akamatsu, L. Gottardi, J. van der Kuur, and B. Jackson, Development of ultra-low-noise tes bolometer arrays, J. Low Temp. Phys. 184, 52 (2016).

[40] N. Poli, J. P. Morten, M. Urech, A. Brataas, D. B. Haviland, and V. Korenivski, Spin Injection and Relaxation in a Mesoscopic Superconductor, Phys. Rev. Lett. 100, 136601 (2008).

[41] N. Beev and M. Kiviranta, Fully differential cryogenic transistor amplifier, Cryogenics 57, 129 (2013).

[42] A. Svilans, M. Leijnse, and H. Linke, Experiments on the thermoelectric properties of quantum dots, C. R. Phys. 17, 1096 (2016). 